\title{
A Multi-method Approach towards Understanding the Pathophysiology of Aortic Dissections - The Complementary Role of In-Silico, In-Vitro and In-Vivo Information
}

\author{
Paula A. Rudenick ${ }^{1}$, Maurizio Bordone ${ }^{2}$, Bart H. Bijnens ${ }^{3}$, Eduardo Soudah ${ }^{2}$, \\ Eugenio Oñate $^{2}$, David Garcia-Dorado ${ }^{4}$, and Arturo Evangelista ${ }^{4}$ \\ ${ }^{1}$ Universitat Pompeu Fabra, Carrer Tanger, 122-140, E-08018 Barcelona, Spain \\ ${ }^{2}$ International Center for Numerical Methods in Engineering (CIMNE), Universitat Politècnica \\ de Catalunya, Barcelona, Spain \\ ${ }^{3}$ ICREA-Universitat Pompeu Fabra, Carrer Tanger, 122-140, E-08018 Barcelona, Spain \\ ${ }^{4}$ University Hospital Vall d'Hebron, Barcelona, Spain
}

\begin{abstract}
Management and follow-up of chronic aortic dissections continues to be a clinical challenge due to progressive aortic dilatation. To predict dilatation, guidelines suggest follow-up of the aortic diameter. However, dilatation is triggered by haemodynamic parameters (pressure and wall shear stresses (WSS)), and geometry of false (FL) and true lumen (TL). We aimed at a better understanding of TL and FL haemodynamics by performing in-silico (CFD) and invitro studies on an idealized dissected aorta and compared this to a typical patient. We observed an increase in diastolic pressure and wall stress in the FL and the presence of diastolic retrograde flow. The inflow jet increased WSS at the proximal FL while a large variability in WSS was induced distally, all being risk factors for wall weakening. In-silico, in-vitro and in-vivo findings were very similar and complementary, showing that their combination can help in a more integrated and extensive assessment of aortic dissections, improving understanding of the haemodynamic conditions and related clinical evolution.
\end{abstract}

Keywords: Aortic dissection, Computational Fluid Dynamics, In-vitro phantoms, Aortic diseases.

\section{Introduction}

Aortic pathologies represent an important subgroup within cardiovascular diseases, and while their prevalence is limited, they are associated with a very high morbidity and mortality ( $>50 \%$ in the acute phase). Despite improved diagnostic and therapeutic techniques, the management and follow-up of aortic dissections continue being a challenge in clinical practice.

Classic aortic dissection is believed to begin with the formation of a tear in the aortic intima that exposes an underlying media layer to the pulsatile pressure of the intraluminal blood (Fig. 1) leading to a longitudinal cleaving of the media layer along the aortic wall, causing the dissection. The dissection process extends typically antegrade (driven by the forward force of the aortic blood flow) but sometimes retrograde from the site of the intimal tear. The lumen will be divided into two parts, the true 
(TL) and the false lumen (FL). In $90 \%$ of cases TL and FL are communicated through entry and exit sites in the dissection flap. The distension of FL during the pulsatile pressure inside the lumina can cause intimal flap movement, distorting the TL shape and narrowing its calibre, potentially leading to TL collapse obstructing side branches and inducing visceral ischemia.

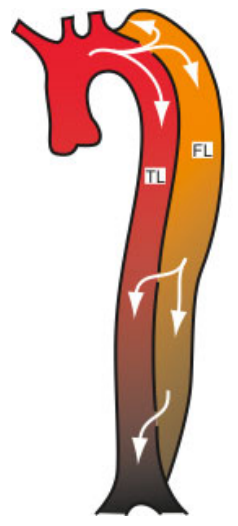

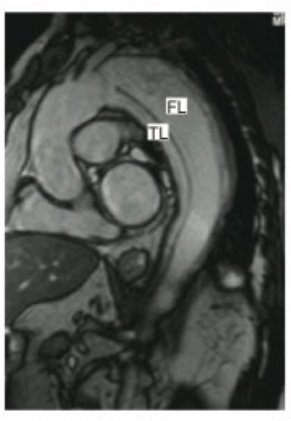

MRI

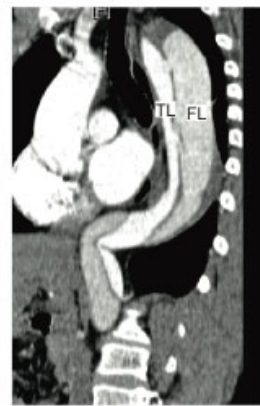

CT
Fig. 1. A classic dissection of the descending aorta (Left). The typical clinical appearance on magnetic resonance (middle) and computed tomography (right) images. TL: True Lumen; FL: False Lumen.

While acute ascending aortic dissections require immediate surgery, descending aortic dissections are often treated medically and persist in the chronic phase [1]. However, these patients still have high mid/long term mortality during the chronic phase, mainly due to the progressive dilatation of the aorta and subsequent rupture.

In current clinical practice, prediction of outcome is mainly based on maximum total aortic diameter, which is compared with guidelines for deciding the best therapeutic approach. However, previous work has shown that maximum diameter is not a reliable determinant of rupture and progression [1-4]. In addition to it, haemodynamic parameters (intra-luminal pressure and flow conditions/wall shear stresses), geometric factors (such as the shape and curvature of the aorta and the communications between
dilatation and risk of rupture.
Whereas an integrated clinical approach towards the biomechanics and haemody-
namics of the dissected aorta is still lacking, based on clinical observations and patient registries, several markers have been suggested to assist in the prediction of

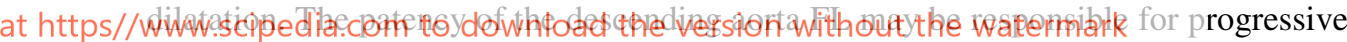
aortic dilation [1] and partial thrombosis of the FL has been found as a predictor of post-discharge mortality in patients with type B acute aortic dissection [5].

It was also observed that prognosis of patients with open communication between TL and FL is poorer than in those without such communication, and free communication with high flow rates carries a higher risk for reoperation because of the high flow pressure and wall stress. Nevertheless, complete obliteration of the FL can occur despite open communication and is possibly related to the size of communication [6]. Poor inflow in the TL and lack of outflow in the FL may have impact in FL dilation and rupture during follow-up period [7].

Therefore, from clinical observations, the importance of tear size and location is clear. However, the contradictory findings on which situations are leading to further 
dilatation of the FL show that there is still a lack of understanding of the interplay of all variables.

Another factor that could affect the dilation of the FL is the compliance or mechanical strength of the dissected aortic wall. Arteries respond to changes in blood pressure and flow conditions by remodelling. Wall shear stress (WSS) is the tangential force resulting from the friction that the flowing blood exerts on the luminal surface. It has been shown that WSS can change the morphology and orientation of the endothelial cell layer [8]. Prolonged high WSS is known to cause vessel dilation and internal elastic lamina fragmentation, and may be the responsible for dissection initiation [9]. On the other hand, inflammatory and atherosclerotic pathways, triggered by low WSS, could also play an important role in dissection pathogenesis. Excessively low WSS could lead to atherosclerotic inflammatory infiltration and thereby cause deterioration of the aortic wall that could lead to mechanical weakening and rupture [10].

Therefore, it is expected that better aortic morphologic and hemodynamic analysis will be much more predictive for aortic dilatation and will improve the clinical stratification of the risk of these patients, facilitating a better therapeutic management.

The aim of this study is to assess whether an integrated approach towards TL and FL haemodynamics will allow us to define risk markers of severe aortic enlargement. For this, in-silico and in vitro studies were performed to investigate the impact of morphological characteristics on the haemodynamics of the TL and FL and the findings were compared to a typical patient from our hospital.

\section{Methods}

\subsection{In-Vivo}

In our hospital, chronic aortic dissection patients undergo regular follow-up with trans-thoracic and trans-oesophageal echocardiography for the quantification of changes in aortic size. Additionally, an MRI study, including short-axis phasecontrast acquisition of blood flow in the distal FL and TL (Fig, 2) is performed and PEDIA

at https//www.scipedia.com to download the version without the watermark

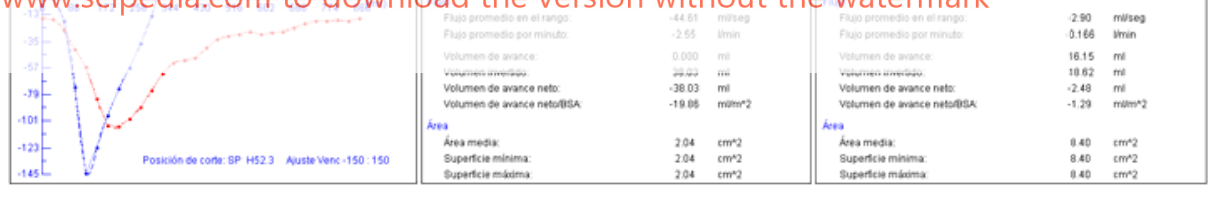

Fig. 2. Typical results from a clinical MRI phase-contrast study. Left: instantaneous volume flow in FL (blue) and TL (red); Middle: measurements in TL; Right: measurements in FL

\subsection{In-Silico}

Idealized geometry. A Computational 3D model of typical type B aortic dissections was constructed with the CAD software GID (CIMNE, Barcelona) (Fig. 3) [11]. The 

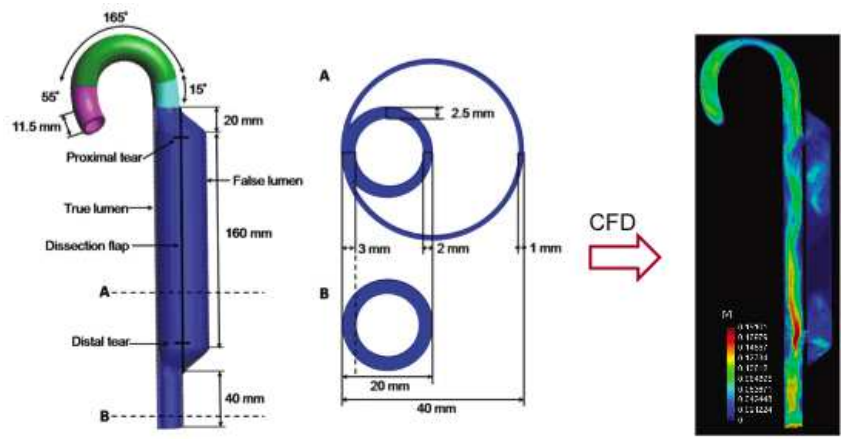

Fig. 3. Left: Idealized geometry of an aortic dissection including the dissected section (A) and the non-dissected aortic section (B); Right: The result of the CFD simulations, showing the velocities at the longitudinal midplane of the dissection.

dimensions of the model, including the aortic arch and the ascending aorta, were selected based on anatomical measurements [12,13]. These are: aortic diameter: $20 \mathrm{~mm}$; dissected segment diameter: $40 \mathrm{~mm}$; FL length: $160 \mathrm{~mm}$; TL thickness: $3 \mathrm{~mm}$; dissection flap thickness: $2 \mathrm{~mm}$; and FL thickness: $1 \mathrm{~mm}$. A proximal and distal tear was included with $10 \mathrm{~mm}$ diameter, corresponding to $25 \%$ of the dissected segment diameter.

Computational fluid dynamics (CFD) simulation. The computational mesh consisted of approximately 1.1 million tetrahedral elements with a size range of $0.5-1.0$ $\mathrm{mm}$ and was created with GID (CIMNE, Barcelona). The CFD simulation was performed using CFD-Tdyn (CompassIS, Barcelona), solving the Navier Stokes equations. The no-slip wall of the dissection model was assumed to be rigid, assuming that in chronic dissection there is reduced flap motion, so that a rigid flap is a good first approximation. Additionally, several studies suggested that the difference in flow induced pressure variations and consequent wall stress between rigid and elastic aortic models is negligible $[14,15]$. Realistic time dependent velocity and pressure waveforms (adapted from [16]) were applied at the inlet and outlet of the fluid domain respectively. We assessed intra-luminal pressure and instantaneous volume flow in

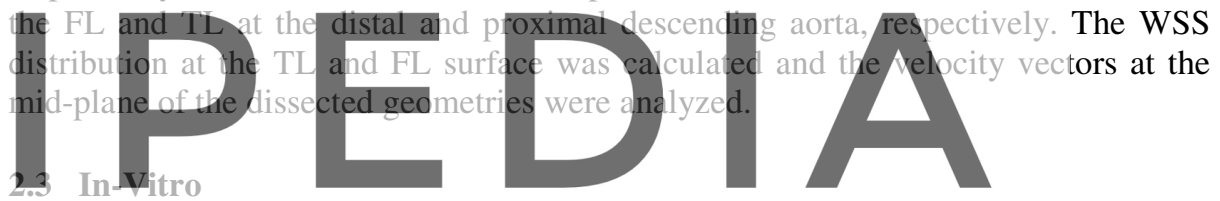

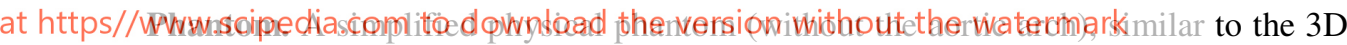
geometry used for the in-silico approach, was made from a compliant and flexible material to meet the tensile strength of the aorta. The model was constructed from two individual parts to simulate the dissection: the TL and the FL. These parts were joined together to form the final model. The TL consisted of a silicone tube of $16 \mathrm{~mm}$ inner diameter and $2 \mathrm{~mm}$ wall thickness in which holes were made to create the tears.

The FL part was custom made by first creating the geometry using modelling clay and PVC tubes. Next, from this, a two-part silicone (RTV) mould is made, which can be used to create multiple wax casts of the FL. Both halves of the mould are held together for casting a replica from beeswax. After solidifying, any mould marks remaining on the wax were carefully polished away. The wax replica was used in a lost-wax technique to create a latex (Kryolan) phantom by dipping the replica in 

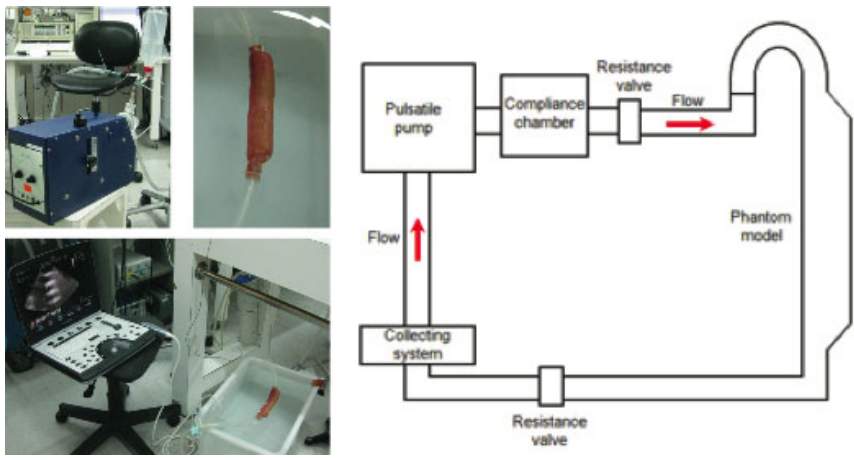

Fig. 4. Experimental set up for the in-vitro measurements. Right: diagram of the circuit. Left: different components of the circuit (top left: pulsatile pump; top right: latex phantom; bottom: portable ultrasound machine and phantom in a water tank).

liquid latex many times at intervals of 1 hour. Once the coating was finished, the model was heated, to remove the wax.

Experimental set up. A dynamic flow circuit, mimicking the human circulatory system, was set up to evaluate flow and morphological characteristics under controlled conditions (Fig. 4). The circuit consisted of a pulsatile pump, a compliance chamber, the dissection model, and a collecting system, connected in series. The flow pump (Harvard Apparatus) was programmed to simulate pulsatile left ventricle output with a heart rate $=70 \mathrm{bpm}$; stroke volume $=70 \mathrm{ml}$; and systolic/diastolic phase ratio $=30 / 70$. Peripheral resistance and systemic pressure were adjusted with the use of resistors (adjustable valves) placed proximal and distal from the phantom.

Measurements/Imaging. TL and FL pressure waveforms were measured with a fluid filled catheter at the distal and proximal sections. Flow was measured using an ultrasonic flow meter (Transonic Systems Inc). Pressure and flow waveforms were digitized using a PowerLab 16/30 with LabChart Pro acquisition and analysis software (ADInstruments, Colorado Springs, CO, USA). Phantom geometry as well as fluid appearance and velocities within the phantom were assessed by two-dimensional and Doppler ultrasotind
GE Healthcare) (Fig
3 Results
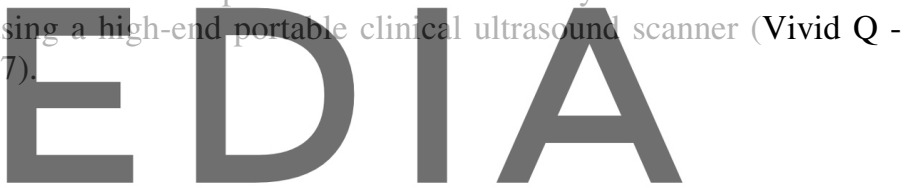

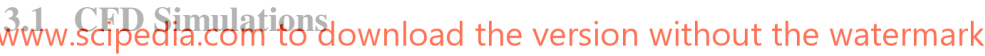

At the distal tear, higher FL pressures were observed at the onset of the cycle, resulting in an antegrade jet through the tear, whereas the FL/TL pressure gradient inverted at the end of the cycle leading to deceleration and inversion of the velocities and resulting in retrograde flow through the distal tear.

Fig. 5 shows the resulting absolute volumetric flows. FL flow variations are remarkably different from the TL, with a biphasic pattern and high early systolic flow.

Fig. 6 shows the normalized velocities during the cycle, obtained at different positions in the model. As can be observed, flow direction in the TL is dominantly antegrade (positive), except at the proximal section were its direction slightly reverses during early diastole. However, in the FL, fluid velocities begin to be retrograde from late systole, resulting in the reverse flow shown in the flow pattern plots (Fig. 7). 

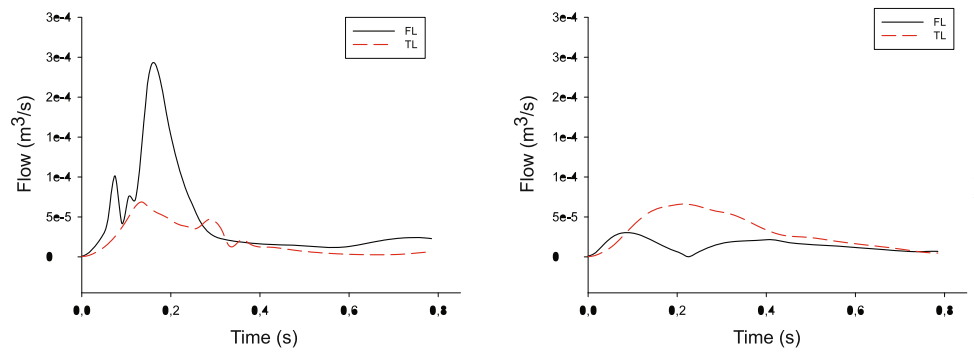

Fig. 5. The instantaneous flow (modulus) in the $\mathrm{TL}$ and $\mathrm{FL}$ at proximal (left) and distal (right) sections

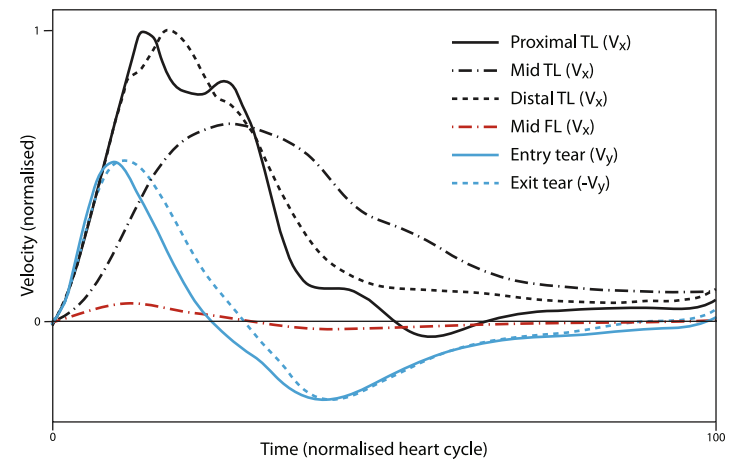

Fig. 6. Normalized mean velocities changes of TL and FL, at different positions of the phantom, and in the entry and exit tears.

Through the entry tear, we can observe a clear inflow during systole and outflow during diastole, while there is outflow during systole and inflow during diastole through the exit tear. The magnitudes of the velocities at both tears are similar. However, there is a shift in the time course indicating the propagation of the fluid wave.

From the assessment of the flow pattern in the dissected region (Fig. 7), we observe a bidirectional flow in the FL with a prominent retrograde during diastole. The most significant elevation in WSS is seen at the impact zone of the entry jet at end-systole, whereas during diastole there was a high variability of WSS in the distal zone.
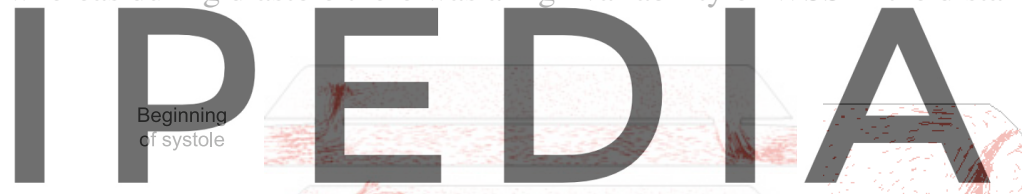

at https//www.scipedfirtelom to download the version without the watermark

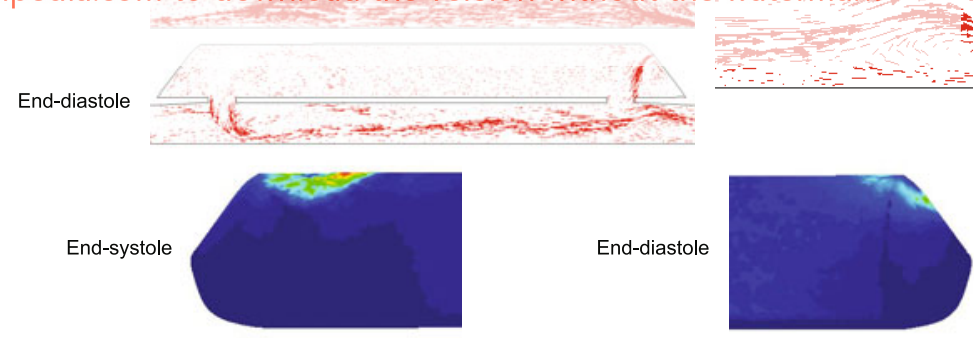

Fig. 7. Top: flow patterns at beginning and end-systole and end-diastole. The zoomed area shows the presence of FL diastolic retrograde flow at the exit tear. Bottom: WSS distributions at the FL surface. Left: the entry tear at end-systole; right: the exit area at end-diastole. 

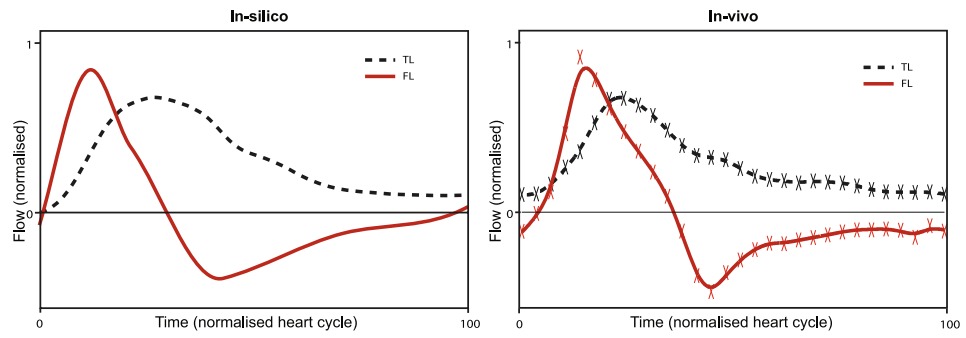

Fig. 10. Comparison of the in-silico instantaneous flow with the measurements from a typical patient

be seen, the observed profiles are remarkably similar, illustrating the usefulness of the in-silico approach to study the haemodynamics of typical patients.

\section{Discussion}

The dilatation of the dissected aorta depends on multiple factors. The cyclic wall stress in the FL is determined by the blood pressure changes, in interaction with the wall properties. Wall properties themselves are related to genetics, chronic pressure levels and flow (in particular WSS).

In our findings, the diastolic pressure in the FL was higher than in the TL, exposing the already weak and thin FL wall to higher wall stress. Additionally, pressures are higher at the distal section than at the proximal section, explaining the distal propagation of dissections.

Complex flow patterns have been thought to increase inflammatory cell infiltration in artery wall, increasing risk rupture $[17,18]$. We show both in-silico and in-vitro that the entry-jet and flow reversals result in complex flow patterns in the FL. A concentrated, jet-like flow is noted, directly impinging on the FL wall at the proximal and distal site during peak systole and diastole, respectively. This fast proximal jet might explain the eccentric dilatation of the proximal FL observed in a subset of patients.
An important hemodynamic factor that infuences vascular remodelling, aortic ex-
pansion and rupture is WSS. WSS influences the morplology and orientation of
endothelial cells [8]. An acute increase in WSS leads to increase of the aortic di-
ameter and weakening of the artic wail because of loss of elastic tissue, change of muscle cell orientation, and acceleration of cell deterioration. On the other hand, the

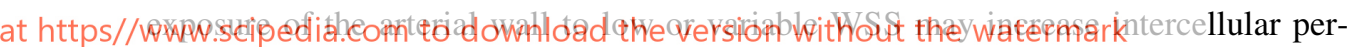
meability and increase the vulnerability of these regions of the vessel to atherosclerosis and weakening that could ultimately lead to rupture.

The cumulative effect of increases in pressure (wall stress) and changes in elastic properties, initiated by altered WSS, results in increased risk of further dilatation and rupture.

Limitations: There are many limitations in both in-silico and in vitro studies of an aortic dissection. We used a flexible dissection phantom to mimic the aortic wall compliance. Despite being an idealized model, its dimensions are based on clinical measurements and this generic model is ideal for parametric studies.

Whereas the overall flow and pressure waveforms were very similar, we had some differences of values between the results obtained with the in-silico and in-vitro models. It was mainly because we compared a flexible physical phantom with a rigid 
2. Bernard, Y., Zimmermann, H., Chocron, S., Litzler, J.-F., Kastler, B., Etievent, J.-P., Meneveau, N., Schiele, F., Bassand, J.-P.: False lumen patency as a predictor of late outcome in aortic dissection. Am. J. Cardiol. 87, 1378-1382 (2001)

3. Nollen, G.J., Groenink, M., Tijssen, J.G., Van Der Wall, E.E., Mulder, B.J.: Aortic stiffness and diameter predict progressive aortic dilatation in patients with Marfan syndrome. Eur. Heart J. 25, 1146-1152 (2004)

4. Neri, E., Barabesi, L., Buklas, D., Vricella, L.A., Benvenuti, A., Tucci, E., Sassi, C., Massetti, M.: Limited role of aortic size in the genesis of acute type A aortic dissection. Eur. J. Cardiothorac. Surg. 28, 857-863 (2005)

5. Tsai, T.T., Evangelista, A., Nienaber, C.A., Myrmel, T., Meinhardt, G., Cooper, J.V., Smith, D.E., Suzuki, T., Fattori, R., Llovet, A., Froehlich, J., Hutchison, S., Distante, A., Sundt, T., Beckman, J., Januzzi Jr., J.L., Isselbacher, E.M., Eagle, K.A.: International Registry of Acute Aortic Dissection: Partial thrombosis of the false lumen in patients with acute type B aortic dissection. N. Engl. J. Med. 357, 349-359 (2007)

6. Erbel, R., Oelert, H., Meyer, J., Puth, M., Mohr-Katoly, S., Hausmann, D., Daniel, W., Maffei, S., Caruso, A., Covino, F.E., et al.: Effect of medical and surgical therapy on aortic dissection evaluated by transesophageal echocardiography. Implications for prognosis and therapy. The European Cooperative Study Group on Echocardiography, Circulation 87, 1604-1615 (1993)

7. Tsai, T.T., Schlicht, M.S., Khanafer, K., Bull, J.L., Valassis, D.T., Williams, D.M., Berguer, R., Eagle, K.A.: Tear size and location impacts false lumen pressure in an ex vivo model of chronic type B aortic dissection. J. Vasc. Surg. 47, 844-851 (2008)

8. Levesque, M.J., Liepsch, D., Moravec, S., Nerem, R.M.: Correlation of endothelial cell shape and wall shear stress in a stenosed dog aorta. Arteriosclerosis 6, 220-229 (1986)

9. Shaaban, A.M., Duerinckx, A.J.: Wall shear stress and early atherosclerosis: a review. AJR Am. J. Roentgenol. 174, 1657-1665 (2000)

10. Malek, A.M., Alper, S.L., Izumo, S.: Hemodynamics shear stress and its role in atherosclerosis. JAMA 282, 2035-2042 (1999)

11. GiD - The personal pre and postprocessor, CIMNE (2006), http://www.gidhome.com/

12. Williams, D.M., LePage, M.A., Lee, D.Y.: The dissected aorta: part I. Early anatomic changes in an in vitro model. Radiology 203, 23-31 (1997)
Strotzer, M., A cebert, H., Lenhart, M. Nitz, W. Wild, T., Manke, \&., Völk, M., Feuerbach,
S.: Morpholosy and hemodynamics in dissegtion of the desconding dotta. Assessment with
MR imaging, Acta Radiol.41, 594-600 (2000) Fluịd structure interaction of patient speçific abdominal aortic aneurysms: a comparison at https//www scipediaccom to dowiload the version withqut the watermark

15. Borghi, A., Wood, N.B., Mohiaddin, R.H., Xu, X.Y.: Fluid-solid interaction simulation of flow and stress pattern in thoracoabdominal aneurysms: a patient-specific study. J. Fluids Struct. 24, 270-280 (2008)

16. Reymond, P., Merenda, F., Perren, F., Rüfenacht, D., Stergiopulos, N.: Validation of a onedimensional model of the systemic arterial tree. Am. J. Physiol. Heart Circ. Physiol. 297, H208-H222 (2009)

17. Gimbrone Jr., M.A., Topper, J.N., Nagel, T., Anderson, K.R., Garcia-Cardeña, G.: Endothelial dysfunction, hemodynamic forces, and atherogenesis. Ann. N Y Acad. Sci. 902, 230-240 (2000)

18. Davies, P.F., Spaan, J.A., Krams, R.: Shear stress biology of the endothelium. Ann. Biomed. Eng. 33, 1714-1718 (2005) 